\title{
An $L$-like model containing very large cardinals
}

\author{
Arthur W. Apter · James Cummings
}

Received: 6 February 2007 / Revised: 20 July 2007 / Published online: 27 May 2008

(C) Springer-Verlag 2008

\begin{abstract}
We force and construct a model in which level by level equivalence between strong compactness and supercompactness holds, along with a strong form of diamond and a version of square consistent with supercompactness. This generalises a result due to the first author. There are no restrictions in our model on the structure of the class of supercompact cardinals.
\end{abstract}

Keywords Supercompact cardinal · Strongly compact cardinal · Diamond . Diamond primed $\cdot$ Diamond star $\cdot$ Diamond plus $\cdot$ Square $\cdot$ Level by level equivalence between strong compactness and supercompactness

Mathematics Subject Classification (2000) $\quad$ 03E35 03 E55

A. W. Apter's research was partially supported by PSC-CUNY Grants and CUNY Collaborative Incentive Grants. J. Cummings's research was partially supported by NSF Grant DMS-0400982.

\section{A. W. Apter}

Department of Mathematics, Baruch College of CUNY, New York, NY 10010, USA

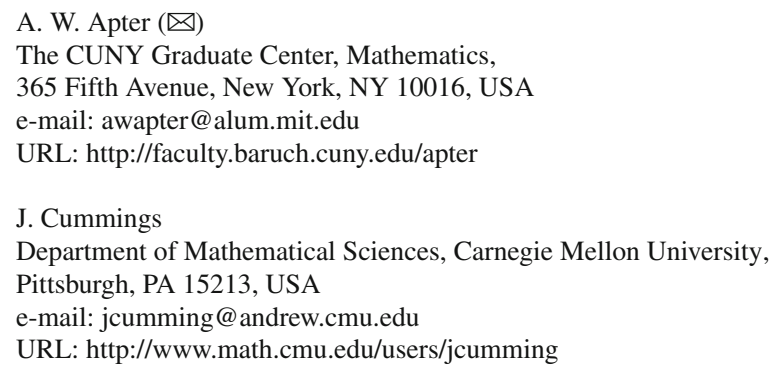




\section{Introduction and preliminaries}

In [1], the first author proved the following theorem.

Theorem 1 Let $V \vDash " Z F C+\mathcal{K} \neq \varnothing$ is the class of supercompact cardinals $+\kappa$ is the least supercompact cardinal". There is then a partial ordering $\mathbb{P} \subseteq V$ such that $V^{\mathbb{P}} \vDash " Z F C+G C H+\mathcal{K}$ is the class of supercompact cardinals (so $\kappa$ is the least supercompact cardinal). In $V^{\mathbb{P}}$, level by level equivalence between strong compactness and supercompactness holds. In addition, in $V^{\mathbb{P}}$, for every $\delta \in A$ where $A$ is a certain stationary subset of $\kappa, \square_{\delta}$ holds, and for every regular uncountable cardinal $\delta, \diamond_{\delta}$ holds".

In terminology used by Woodin, this theorem can be classified as an "inner model theorem proven via forcing." This is since the model constructed satisfies pleasant properties one usually associates with an inner model, namely GCH and many instances of square and diamond, along with a property one might perhaps expect if a "nice" inner model containing supercompact cardinals ever were to be constructed, namely level by level equivalence between strong compactness and supercompactness.

The purpose of this paper is to extend and generalise Theorem 1, in order to construct a model for level by level equivalence between strong compactness and supercompactness in which a version of square consistent with supercompactness holds on the class of all infinite cardinals and in which a strong form of diamond holds on a proper class of regular cardinals. Our model for level by level equivalence between strong compactness and supercompactness consequently becomes, in a sense, even more "inner model like" than the one for Theorem 1. Specifically, we prove the following theorem.

Theorem 2 Let $V \vDash " Z F C+G C H+\mathcal{K} \neq \varnothing$ is the class of supercompact cardinals". There is then a partial ordering $\mathbb{P} \subseteq V$ such that $V^{\mathbb{P}} \vDash " Z F C+G C H+\mathcal{K}$ is the class of supercompact cardinals + Level by level equivalence between strong compactness and supercompactness holds". In $V^{\mathbb{P}}, \square_{\gamma}^{S}$ holds for every infinite cardinal $\gamma$, where $S=\operatorname{Safe}(\gamma)$. In addition, in $V^{\mathbb{P}}, \diamond_{\mu}$ holds for every $\mu$ which is inaccessible or the successor of a singular cardinal, and $\diamond_{\mu}^{+}$holds for every $\mu$ which is the successor of a regular cardinal.

Pertinent definitions are presented at various junctures throughout the course of the paper. In particular, we will give the definitions of $S=\operatorname{Safe}(\gamma)$ (Definition 2.2) and $\square_{\gamma}^{S}$ in Sect. 2, and the definitions of our various diamond principles in Sect. 3. We do, however, take this opportunity to mention that for $\kappa$ a regular cardinal and $\alpha$ an ordinal, $\operatorname{Add}(\kappa, \alpha)$ is the standard Cohen poset for adding $\alpha$ many new subsets of $\kappa$.

The overall structure of this paper is as follows. In Sect. 1, we provide a brief introduction. In Sect. 2, we discuss forcing the relevant version of square. In Sect. 3, we discuss forcing a strong form of diamond. In Sect. 4, we give a proof of Theorem 2.

Before continuing, we do wish now to take the opportunity to state a result which will be used in the proof of Theorem 2. This is a corollary of Theorems 3 and 31 and Corollary 14 of Hamkins' paper [8]. This theorem is a generalisation of Hamkins' Gap Forcing Theorem and Corollary 16 of [9] and [10] (and we refer readers to [8-10] for further details). We therefore state the theorem we will be using now, along with some 
associated terminology. Suppose $\mathbb{P}$ is a partial ordering which can be written as $\mathbb{Q} * \dot{\mathbb{R}}$, where $|\mathbb{Q}| \leq \delta, \mathbb{Q}$ is nontrivial, and $\vdash_{\mathbb{Q}}$ " $\mathbb{R}$ is $(\delta+1)$-strategically closed" (meaning that there is a winning strategy for player II in the game having length $\delta+1$ ). In Hamkins' terminology of [8], $\mathbb{P}$ admits a closure point at $\delta$. In Hamkins' terminology of [9] and [10], $\mathbb{P}$ is mild with respect to a cardinal $\kappa$ iff every set of ordinals $x$ in $V^{\mathbb{P}}$ of size below $\kappa$ has a "nice" name $\tau$ in $V$ of size below $\kappa$, i.e., there is a set $y$ in $V$, $|y|<\kappa$, such that any ordinal forced by a condition in $\mathbb{P}$ to be in $\tau$ is an element of $y$. Also, as in the terminology of [8-10], and elsewhere, an embedding $j: \bar{V} \rightarrow \bar{M}$ is amenable to $\bar{V}$ when $j \uparrow A \in \bar{V}$ for any $A \in \bar{V}$. The specific corollary of Theorems 3 and 31 and Corollary 14 of [8] we will be using is then the following.

Theorem 3 (Hamkins) Suppose that $V[G]$ is a forcing extension obtained by forcing that admits a closure point at some regular $\delta<\kappa$. Suppose further that $j: V[G] \rightarrow$ $M[j(G)]$ is an embedding with critical point $\kappa$ for which $M[j(G)] \subseteq V[G]$ and $M[j(G)]^{\delta} \subseteq M[j(G)]$ in $V[G]$. Then $M \subseteq V$; indeed, $M=V \cap M[j(G)]$. If the full embedding $j$ is amenable to $V[G]$, then the restricted embedding $j \uparrow V: V \rightarrow M$ is amenable to $V$. If $j$ is definable from parameters (such as a measure or extender) in $V[G]$, then the restricted embedding $j \uparrow V$ is definable from the names of those parameters in $V$. Finally, if $\mathbb{P}$ is mild with respect to $\kappa$ and $\kappa$ is $\lambda$-strongly compact in $V[G]$ for any $\lambda \geq \kappa$, then $\kappa$ is $\lambda$-strongly compact in $V$.

It immediately follows from Theorem 3 that any cardinal $\kappa$ which is $\lambda$-supercompact in a generic extension obtained by forcing that admits a closure point below $\kappa$ (such as at $\omega$ ) must also be $\lambda$-supercompact in the ground model. In particular, if $\bar{V}$ is a forcing extension of $V$ by a poset that admits a closure point at $\omega$ in which each supercompact cardinal is preserved, the class of supercompact cardinals in $\bar{V}$ remains the same as in $V$.

We conclude Sect. 1 with a short discussion of some important terminology. Suppose $V$ is a model of ZFC in which for all regular cardinals $\kappa<\lambda, \kappa$ is $\lambda$-strongly compact iff $\kappa$ is $\lambda$-supercompact, except possibly if $\kappa$ is a measurable limit of cardinals $\delta$ which are $\lambda$-supercompact. Such a model will be said to witness level by level equivalence between strong compactness and supercompactness. We will also say that $\kappa$ is a witness to level by level equivalence between strong compactness and supercompactness iff for every regular cardinal $\lambda>\kappa, \kappa$ is $\lambda$-strongly compact iff $\kappa$ is $\lambda$-supercompact. Note that the exception is provided by a theorem of Menas [13], who showed that if $\kappa$ is a measurable limit of cardinals $\delta$ which are $\lambda$-strongly compact, then $\kappa$ is $\lambda$-strongly compact but need not be $\lambda$-supercompact. Models in which level by level equivalence between strong compactness and supercompactness holds nontrivially were first constructed in [3].

\section{Forcing a weak version of $\square$}

\subsection{Partial squares and the basic forcing}

We state a partial version of $\square$ compatible with supercompact cardinals. Square sequences of this kind were first shown to be consistent with supercompactness by 
Foreman and Magidor [7, p. 191], using techniques of Baumgartner. In the notation of Definition 2.1 they showed that $\square_{\kappa^{+\omega}}^{\left\{\kappa^{+n}: n<\omega\right\}}$ is consistent with $\kappa$ being supercompact.

Given a set $S$ of regular cardinals, we denote by $\operatorname{cof}(S)$ the class of ordinals $\alpha$ such that $\operatorname{cf}(\alpha) \in S$.

Definition 2.1 Let $\gamma$ be an infinite cardinal and let $S$ be a set of regular cardinals which are less than or equal to $\gamma$. Then a $\square_{\gamma}^{S}$-sequence is a sequence $\left\langle C_{\alpha}: \alpha \in \gamma^{+} \cap \operatorname{cof}(S)\right\rangle$ such that

1. $C_{\alpha}$ is club in $\alpha$ and ot $\left(C_{\alpha}\right) \leq \gamma$.

2. If $\beta \in \lim \left(C_{\alpha}\right) \cap \lim \left(C_{\alpha^{\prime}}\right)$ then $C_{\alpha} \cap \beta=C_{\alpha^{\prime}} \cap \beta$.

$\square_{\gamma}^{S}$ holds if and only if there is a $\square_{\gamma}^{S}$-sequence.

We note that if $T=\left(\gamma^{+} \cap \operatorname{cof}(S)\right) \cup\left\{\eta: \exists \beta \in \gamma^{+} \cap \operatorname{cof}(S) \eta \in \lim \left(C_{\beta}\right)\right\}$ then we can trivially extend the domain of the sequence to $T$, by defining $C_{\eta}=C_{\beta} \cap \eta$ for some (any) $\beta$ with $\eta \in \lim \left(C_{\beta}\right)$.

We define a forcing poset $\mathbb{Q}^{\square}(\gamma, S)$ to add such a sequence. Elements of $\mathbb{Q}^{\square}(\gamma, S)$ are all pairs $(q, \eta)$ such that $\eta<\gamma^{+}$, and $q$ is a partial function on $\gamma^{+}$such that

1. $(\eta+1) \cap \operatorname{cof}(S) \subseteq \operatorname{dom}(q) \subseteq \eta+1$.

2. For all $\alpha \in \operatorname{dom}(q)$

(a) $q(\alpha)$ is club in $\alpha$, and ot $(q(\alpha)) \leq \gamma$.

(b) For all $\beta \in \lim q(\alpha), \beta \in \operatorname{dom}(q)$ and $q(\alpha) \cap \beta=q(\beta)$.

$(q, \theta)$ extends $(p, \eta)$ if and only if $\theta \geq \eta, \operatorname{dom}(q) \cap(\eta+1)=\operatorname{dom}(p)$ and $q \uparrow$ $\operatorname{dom}(p)=p$.

Routine arguments [4, Lemma 6.1] show that the poset is $(\gamma+1)$-strategically closed, in particular it adds no $\gamma$-sequences. If $2^{\gamma}=\gamma^{+}$then the forcing contains only $\gamma^{+}$many conditions, so trivially has $\gamma^{++}$-cc. The key property is that when we form the union of a decreasing chain of conditions of length less than $\min (S)$, we can obtain a condition, because we are only obliged to put points of $\operatorname{cof}(S)$ into the support of a condition.

Definition 2.2 For each infinite cardinal $\gamma$, a regular cardinal $\mu$ is safe for $\gamma$ if and only if

1. $\mu \leq \gamma$.

2. For every cardinal $\lambda \leq \gamma$, if $\lambda$ is $\gamma^{+}$-supercompact then $\lambda \leq \mu$.

$\operatorname{Safe}(\gamma)$ is the set of safe regular cardinals for $\gamma$.

We note that the safe set is a final segment of $\operatorname{REG} \cap(\gamma+1)$, and that the safe set can only be empty when $\gamma$ is a singular limit of cardinals which are $\gamma^{+}$-supercompact. In addition, by the remarks immediately following the statement of Theorem $3, \operatorname{Safe}(\gamma)$ is upwards absolute to any cardinal and cofinality preserving forcing extension by a poset admitting a closure point at $\omega$.

The following easy lemma (essentially due to Solovay) motivates the definition of the safe set.

Lemma 2.3 If $S$ is a final segment of $\operatorname{REG} \cap(\gamma+1)$ and $\square_{\gamma}^{S}$ holds then $S \subseteq \operatorname{Safe}(\gamma)$. 
Proof Suppose not, then by definition there is $\mu \in S$ such that $\mu<\lambda \leq \gamma$ and $\lambda$ is $\gamma^{+}$-supercompact. Fix a sequence $\left\langle C_{\alpha}: \alpha \in \gamma^{+} \cap \operatorname{cof}(S)\right\rangle$ witnessing the square.

In particular $C_{\alpha}$ is defined for every $\alpha$ in the stationary set $\gamma^{+} \cap \operatorname{cof}(S)$, and ot $\left(C_{\alpha}\right) \leq \gamma$, so by Fodor's lemma we may find a stationary set $T \subseteq \gamma^{+} \cap \operatorname{cof}(S)$ and an ordinal $\eta$ such that ot $\left(C_{\alpha}\right)=\eta$ for all $\alpha \in T$. It follows easily from the coherence of the clubs $C_{\beta}$ that $T \cap \lim \left(C_{\beta}\right)$ has size at most 1 for all $\beta \in \gamma^{+} \cap \operatorname{cof}(S)$. In particular $T$ cannot reflect at any point of $\gamma^{+} \cap \operatorname{cof}(S)$.

But the $\gamma^{+}$-strong compactness of $\lambda$ [14, Theorem 4.8] implies that $T$ must reflect at some point $\zeta$ with $\mu<\operatorname{cf}(\zeta)<\gamma$. This is a contradiction since $\operatorname{cf}(\zeta) \in S$.

\subsection{Iteration and preservation}

We assume that $V$ satisfies GCH. In the cases of interest $V$ will contain some supercompact cardinals.

We define an Easton support iteration $\mathbb{P}_{\infty}^{\square}$ of length ON (the superscript is intended to distinguish this iteration of length $\infty$ from some other ones appearing later in the paper). As usual $\mathbb{P}_{\gamma}$ is the forcing up to stage $\gamma$ and $\dot{\mathbb{Q}}_{\gamma}^{\square}$ is a $\mathbb{P}_{\gamma}^{\square}$-name for the forcing poset to be used at stage $\gamma \cdot \mathbb{Q}_{\gamma}^{\square}$ is trivial unless $\gamma$ is zero or an infinite cardinal, and $\mathbb{Q}_{0}^{\square}=\operatorname{Add}(\omega, 1)$. At each stage $\gamma$ where $\gamma$ is an infinite cardinal, we will set $\mathbb{Q}_{\gamma}^{\square}=\mathbb{Q}^{\square}(\gamma, S)^{V^{\mathbb{P}} \square}$ where $S=\operatorname{Safe}(\gamma)^{V}$ and $\mathbb{Q}^{\square}(\gamma, S)$ is the forcing poset defined in Sect. 2.1. At all other stages, the forcing is trivial. Routine arguments show that this iteration preserves all cardinals and cofinalities, together with $\mathrm{GCH}$ and the fact that $V^{\mathbb{P}} \square$ is a model of ZFC. In addition, it is easily verified that for every infinite cardinal $\gamma, \square_{\gamma}^{S}$ holds in $V^{\mathbb{P}_{\infty}^{\square}}$.

As we mentioned above, the following result is a descendant of results by Foreman and Magidor [7, p. 191]. The trick in the master condition argument first appeared in unpublished work of Baumgartner.

Theorem 4 If $\kappa$ is supercompact in $V$, then the supercompactness of $\kappa$ is preserved in the extension by $\mathbb{P}_{\infty}^{\square}$.

Proof Let $\gamma>\kappa$ be regular and let $U$ be a supercompactness measure on $P_{\kappa} \gamma$. Let $j: V \rightarrow M$ be the ultrapower map. As usual $\operatorname{crit}(j)=\kappa$, and $\gamma^{+}<j(\kappa)<j(\gamma)<$ $\gamma^{++}$.

It will suffice to show that $\kappa$ is $\gamma$-supercompact in the extension by $\mathbb{P}_{\gamma+1}^{\square}$, since the rest of the iteration adds no new subsets of $P_{\kappa} \gamma$. As usual the resemblance between $V$ and $M$ implies that $\mathbb{P}_{\gamma+1}^{\square}$ is an initial segment of $j\left(\mathbb{P}_{\gamma+1}^{\square}\right)$.

We will break up the generic object for $\mathbb{P}_{\gamma+1}^{\square}$ as $G * g * H$ where $G$ is generic for $\mathbb{P}_{\kappa}^{\square}, g$ is generic for the part of the iteration in the interval $[\kappa, \gamma)$, and $H$ is generic for the forcing at $\gamma$. It is easy to see that $\mathbb{P}_{\gamma}^{\square}$ has cardinality at most $\gamma$, so in particular $V[G * g] \vDash \gamma M[G * g] \subseteq M[G * g]$.

Let $\mathbb{R} \in M[G * g * H]$ be the usual forcing for prolonging $G * g * H$ to a generic filter for $j\left(\mathbb{P}_{\kappa}^{\square}\right)$. By the usual arguments, from the point of view of $V[G * g * H]$ this poset 
has cardinality $\gamma^{+}$and is $\gamma^{+}$-closed, so we may build a generic filter $h \in V[G * g * H]$ for it and extend $j$ to get $j: V[G] \rightarrow M[j(G)]$, where $j(G)=G * g * H * h$.

We note that since $\mathbb{R}$ is sufficiently closed, $V[G * g * H] \vDash \gamma M[j(G)] \subseteq M[j(G)]$. Let $\mathbb{S} \in M[j(G)]$ be the natural forcing for prolonging $j(G)$ to a $j\left(\mathbb{P}_{\gamma}^{\square}\right)$-generic filter. Again $\mathbb{S}$ has cardinality $\gamma^{+}$and is $\gamma^{+}$-closed from the point of view of $V[G * g * H]$, but to lift the embedding we need a generic filter which contains $j$ “ $g$.

We will build a suitable master condition. Let $\delta$ be such that in $M, \delta$ is a cardinal with $j(\kappa) \leq \delta<j(\gamma)$. The key point is that by elementarity $j(\kappa)$ is $j(\gamma)$-supercompact in $M$, in particular $j(\kappa)$ is $\delta^{+}$-supercompact: so the set $\operatorname{Safe}^{M}(\delta)$ contains only cardinals greater than or equal to $j(\kappa)$, and in particular since $\gamma<j(\kappa)$ we have $\gamma<\min \left(\operatorname{Safe}^{M}(\delta)\right)$.

Now we consider the partial function $r$ defined as follows: the domain of $r$ is $\bigcup_{s \in g} \operatorname{dom}(j(s))$, and for each $\delta \in \operatorname{dom}(r), r(\delta)=\bigcup_{s \in g} j(s)(\delta)$. We claim that this is a condition in $\mathbb{S}$. The key point is that $|g| \leq \gamma<\min \left(\operatorname{Safe}^{M}(\delta)\right)$, so that $r(\delta)$ names the union of a rather short chain of conditions and hence is a name for a condition. So we may lift $j$ once again to obtain $j: V[G * g] \rightarrow M[j(G * g)]$.

To finish we just note that $\mathbb{Q}_{\gamma}^{\square}$ adds no $\gamma$-sequences and $\left|P_{\kappa} \gamma\right|=\gamma$. We may therefore transfer the generic object $H$ along $j$ to obtain $j: V[G * g * H] \rightarrow$ $M[j(G * g * H)]$.

\section{Forcing $\diamond_{\lambda^{+}}^{+}$}

\subsection{Strong diamond and the basic forcing}

We recall that

1. $\diamond_{\lambda^{+}}^{\prime}$ is the assertion that there exists a sequence $\left\langle\mathcal{S}_{\alpha}: \alpha<\lambda^{+}\right\rangle$such that

(a) For every $\alpha, \mathcal{S}_{\alpha}$ is a family of subsets of $\alpha$ with $\left|\mathcal{S}_{\alpha}\right| \leq \lambda$.

(b) For every $X \subseteq \lambda^{+}$, the set $\left\{\alpha<\lambda^{+}: X \cap \alpha \in \mathcal{S}_{\alpha}\right\}$ is stationary in $\alpha$.

2. $\diamond_{\lambda^{+}}^{*}$ is the assertion that there exists a sequence $\left\langle\mathcal{S}_{\alpha}: \alpha<\lambda^{+}\right\rangle$such that

(a) For every $\alpha, \mathcal{S}_{\alpha}$ is a family of subsets of $\alpha$ with $\left|\mathcal{S}_{\alpha}\right| \leq \lambda$.

(b) For every $X \subseteq \lambda^{+}$, there is $C \subseteq \lambda^{+}$a club set such that $\forall \alpha \in C X \cap \alpha \in \mathcal{S}_{\alpha}$.

3. $\diamond_{\lambda^{+}}^{+}$is the assertion that there exists a sequence $\left\langle\mathcal{S}_{\alpha}: \alpha<\lambda^{+}\right\rangle$such that

(a) For every $\alpha, \mathcal{S}_{\alpha}$ is a family of subsets of $\alpha$ with $\left|\mathcal{S}_{\alpha}\right| \leq \lambda$.

(b) For every $X \subseteq \lambda^{+}$, there is $C \subseteq \lambda^{+}$a club set such $\forall \alpha \in C X \cap \alpha, C \cap \alpha \in \mathcal{S}_{\alpha}$.

Kunen [6, Theorem 2] showed that $\diamond_{\lambda^{+}}^{\prime}$ is equivalent to $\diamond_{\lambda^{+}}$. In unpublished work Jensen showed that in general $\diamond_{\lambda^{+}}^{*}$ is stronger than $\diamond_{\lambda^{+}}$and $\diamond_{\lambda^{+}}^{+}$is stronger than $\diamond_{\lambda^{+}}^{*}$.

Jensen showed [5] that $\diamond_{\lambda^{+}}^{+}$holds in $L$, and that a $\diamond_{\omega_{1}}^{*}$-sequence can be added by countably closed forcing [6, Lemma 8.3] when $\lambda=\omega$. It is probably possible to adapt that argument to show that a $\diamond_{\lambda^{+}}^{+}$-sequence can be added by $\lambda^{+}$-directedclosed forcing; it is not clear to us whether such an adapted poset would work for our results, since we will be preserving large cardinals by something more elaborate than a straightforward master condition argument.

We will use a poset constructed by Cummings, Foreman and Magidor (see [4, Sect. 12]). We give a fairly detailed exposition here to make this paper reasonably 
self-contained, and to stress that there is some extra flexibility in computing lower bounds in the poset which will be useful later.

We fix $\lambda$ a cardinal with $2^{\lambda}=\lambda^{+}$. We will define a poset $\mathbb{Q}^{\diamond}\left(\lambda^{+}\right)$such that $\mathbb{Q}^{\diamond}\left(\lambda^{+}\right)$ adds $\diamond_{\lambda^{+}}^{+}$, where $\mathbb{Q}^{\diamond}\left(\lambda^{+}\right)$is $\lambda^{+}$-directed closed and $\lambda^{++}$-cc.

The main idea is that we will add a $\diamond_{\lambda^{+}}^{\prime}$-sequence, and then iterate in length $\lambda^{++}$ by adding club subsets of $\lambda^{+}$so as to make this sequence into a $\diamond_{\lambda^{+}}^{+}$-sequence. We will then let $\mathbb{Q}^{\diamond}\left(\lambda^{+}\right)$be the result $\mathbb{P}_{\lambda^{++}}$of this iteration, which will turn out to have $\lambda^{++}$-cc.

We start by defining a poset $\mathbb{Q}_{0}$ to add the $\diamond_{\lambda^{+}}^{\prime}$-sequence. $\mathbb{Q}_{0}$ is the set of those $q$ such that $q=\left\langle\mathcal{S}_{\alpha}: \alpha \leq \beta\right\rangle$ where

1. $\beta<\lambda^{+}$.

2. For every $\alpha \leq \beta$

(a) $\mathcal{S}_{\alpha}$ is a family of subsets of $\alpha$.

(b) $\left|\mathcal{S}_{\alpha}\right| \leq \lambda$.

The ordering is by end-extension. One can check by standard arguments that $\diamond_{\lambda^{+}}^{\prime}$ holds in the extension by $\mathbb{Q}_{0}$, but we will not do this since it follows from our later analysis.

For $\alpha>0$ we will choose (by some bookkeeping scheme) $\dot{X}_{\alpha}$ a $\mathbb{P}_{\alpha}$-name for a subset of $\lambda^{+}$, and then define $\mathbb{Q}_{\alpha}$ in $V^{\mathbb{P}_{\alpha}}$ to be the set of $c$ such that

1. $c$ is closed and bounded in $\lambda^{+}$.

2. $\forall \beta \in \lim (c) X_{\alpha} \cap \beta, c \cap \beta \in \mathcal{S}_{\beta}$.

Here $X_{\alpha}$ is the realisation of the term $\dot{X}_{\alpha}$, and $\left\langle\mathcal{S}_{\alpha}: \alpha<\lambda^{+}\right\rangle$is the $\diamond_{\lambda^{+}}^{\prime}$-sequence added by $\mathbb{Q}_{0}$. The ordering is end-extension. The bookkeeping will arrange that after $\lambda^{++}$steps in the $\lambda^{++}$-cc iteration we have handled every subset of $\lambda^{+}$.

To complete the definition of our iteration, we specify that we will force with supports of size at most $\lambda$; equivalently we will form inverse limits at limit stages $\delta$ with $\operatorname{cf}(\delta) \leq \lambda$, and direct limits when $\operatorname{cf}(\delta)>\lambda$. As usual when we are iterating forcing to shoot club sets, the key point is to prove that there is a dense set of "tame" conditions.

Definition 3.1 A condition $p \in \mathbb{P}_{\alpha}$ is rectangular if and only if there is a limit ordinal $\beta<\lambda^{+}$such that

1. $p(0)$ has the form $\left\langle\mathcal{S}_{\gamma}: \gamma \leq \beta\right\rangle$.

2. For all $\eta \in \operatorname{supp}(p)$ with $\eta>0$

(a) There exist $d_{\eta}, x_{\eta} \in V$ such that $p\left\lceil\eta \Vdash\right.$ “ $p(\eta)=\check{d}_{\eta}, \dot{X}_{\eta} \cap \beta=\check{x}_{\eta}$ ".

(b) $\max \left(d_{\eta}\right)=\beta, \beta \in \lim \left(d_{\eta}\right)$.

Since $p$ is a condition, it follows that $x_{\eta}, d_{\eta} \cap \beta \in \mathcal{S}_{\beta}$.

In a harmless abuse of notation we will often assume that for $p$ rectangular, $p(\gamma)$ is literally a canonical name for an element of $V$. We call the ordinal $\beta$ the height of $p$. Let $\mathbb{P}_{\alpha}^{\text {rect }}$ be the set of rectangular conditions in $\mathbb{P}_{\alpha}$.

Lemma 3.2 Let $1 \leq \alpha \leq \lambda^{++}$. Then 
1. $\mathbb{P}_{\alpha}^{\text {rect }}$ is $\lambda^{+}$-directed closed. Moreover, if $\left\{p_{\gamma}: \gamma<\mu\right\}$ is a directed set of rectangular conditions for some $\mu<\lambda^{+}$, and the height of $p_{\gamma}$ is $\sigma_{\gamma}$, then there is a greatest lower bound (in $\mathbb{P}_{\alpha}^{\text {rect }}$ ) $p$ which is given by

(a) $\operatorname{supp}(p)=\bigcup_{\gamma<\mu} \operatorname{supp}\left(p_{\gamma}\right)$.

(b) $\operatorname{dom}(p(0))=\sigma+1$, where $\sigma=\sup _{\gamma<\mu} \sigma_{\gamma}$.

(c) $p(0) \uparrow \sigma=\bigcup_{\gamma<\mu} p_{\gamma}(0)$.

(d) For $\beta>0, p(\beta)=\bigcup_{\gamma<\mu}\left\{p_{\gamma}(\beta): \beta \in \operatorname{dom}\left(p_{\gamma}\right)\right\} \cup\{\sigma\}$.

(e) $p(0)(\sigma)=\{p(\beta) \cap \sigma: \beta \in \operatorname{dom}(p), \beta>0\} \cup\left\{x_{\beta}: \beta \in \operatorname{dom}(p), \beta>0\right\}$, where $x_{\beta}$ is the subset of $\sigma$ such that $p \nmid \beta \Vdash$ " $\dot{X}_{\beta} \cap \sigma=\check{x}_{\beta}$ ".

$p \in \mathbb{P}_{\alpha}^{\text {rect }}$ and $p$ has height $\sigma$.

2. $\mathbb{P}_{\alpha}^{\text {rect }}$ is dense in $\mathbb{P}_{\alpha}$.

Proof The first claim is easy to verify. We prove the second claim by induction on $\alpha$. Suppose $\mathbb{P}_{\bar{\alpha}}^{\text {rect }}$ is dense in $\mathbb{P}_{\bar{\alpha}}$ for all $\bar{\alpha}<\alpha$. We show $\mathbb{P}_{\alpha}^{\text {rect }}$ is dense in $\mathbb{P}_{\alpha}$.

Case $1 \alpha=1, \mathbb{P}_{1}^{\text {rect }}=\mathbb{P}_{1} \simeq \mathbb{Q}_{0}$ and there is nothing to prove.

Case $2 \alpha=\beta+1$. Fix a condition $p \in \mathbb{P}_{\beta+1}^{\text {rect }}$.

By induction, we know that $\mathbb{P}_{\beta}^{\text {rect }}$ is $\lambda^{+}$-closed and dense in $\mathbb{P}_{\beta}$. In particular $\mathbb{P}_{\beta}$ adds no bounded subsets of $\lambda^{+}$, and so we may choose $p_{0} \leq p \uparrow \beta$ such that $p_{0}$ decides the value of $p(\beta)$, say that $p_{0}$ forces $p(\beta)$ to equal $c$ where $\max (c)=\gamma$. As $\mathbb{P}_{\beta}^{\text {rect }}$ is dense, we may choose $p_{0} \in \mathbb{P}_{\beta}^{\text {rect }}$ and by extending if necessary may also assume that the height of $p_{0}$ is greater than $\gamma$.

Now we argue in a similar vein to build a decreasing $\omega$-sequence of conditions $p_{0}>p_{1}>p_{2}>\ldots$ and an increasing $\omega$-sequence of ordinals $\rho_{0}<\rho_{1}<\ldots$, where $p_{n}$ is a condition in $\mathbb{P}_{\beta}^{\text {rect }}$ of height $\rho_{n}$, and $p_{n+1}$ decides $\dot{X}_{\beta} \cap \rho_{n}$; say $p_{n+1} \Vdash$ " $\dot{X}_{\beta} \cap \rho_{n}=x_{n}$ ". By the first claim of the Lemma, we may form a greatest lower bound for $\vec{p}$ which will be a condition $q \in \mathbb{P}_{\beta}^{\text {rect }}$ of height $\rho=\sup \rho_{n}$.

Now we define $q^{+}$as follows. $\operatorname{dom}\left(q^{+}\right)=\beta+1$, and $q^{+}(\gamma)=q(\gamma)$ for $0<$ $\gamma<\beta \cdot q^{+}(\beta)=c \cup\left\{\rho_{0}, \rho_{1}, \ldots, \rho\right\} \cdot q^{+}(0)\left\lceil\rho=q(0) \uparrow \rho\right.$, and $q^{+}(0)(\rho)=$ $q(0)(\rho) \cup\left\{q^{+}(\beta), \cup_{n} x_{n}\right\}$. It is now routine to check that $q^{+} \in \mathbb{P}_{\beta+1}, q^{+}$is rectangular of height $\rho$, and $q^{+}$refines $p$.

Case $3 \alpha$ is limit with $\operatorname{cf}(\alpha) \geq \lambda^{+}$. Fix $p \in \mathbb{P}_{\alpha}$, then the support of $p$ is bounded by some $\beta<\alpha$. By induction we may find $q \leq p \uparrow \beta$ with $q \in \mathbb{P}_{\beta}^{\text {rect }}$; if $q^{+} \in \mathbb{P}_{\alpha}$ is defined by $q^{+} \uparrow \beta=q$ and $q^{+} \uparrow[\beta, \alpha)=1$ then $q^{+} \in \mathbb{P}_{\alpha}^{\text {rect }}$ and $q^{+} \leq p$, as required.

Case $4 \alpha$ is limit and $\operatorname{cf}(\alpha) \leq \lambda$. Choose a sequence $\left\langle\alpha_{i}: i<\operatorname{cf}(\alpha)\right\rangle$ which is increasing, continuous and cofinal in $\alpha$. Fix $p \in \mathbb{P}_{\alpha}$.

We will define a decreasing sequence of conditions $\left\langle p_{i}: i \leq \operatorname{cf}(\alpha)\right\rangle$ such that $p_{0} \leq p, p_{i}\left\lceil\alpha_{i} \in \mathbb{P}_{\alpha_{i}}^{\text {rect }}\right.$ for each $i$, and $p_{\mathrm{cf}(\alpha)} \in \mathbb{P}_{\alpha}^{\text {rect }}$. We let $\sigma_{i}$ denote the height of $p_{i} \uparrow \alpha_{i}$

$i=0$. Let $q_{0} \leq p\left\lceil\alpha_{0}, q_{0} \in \mathbb{P}_{\alpha_{0}}^{\text {rect }}\right.$. Let $p_{0}\left\lceil\alpha_{0}=q_{0}, p_{0} \uparrow\left[\alpha_{0}, \alpha\right)=p\left\lceil\left[\alpha_{0}, \alpha\right)\right.\right.$.

$i=j+1$. Let $q_{i} \leq p_{j}\left\lceil\alpha_{i}, q_{i} \in \mathbb{P}_{\alpha_{i}}^{\text {rect }}\right.$. Now let $p_{i}\left\lceil\alpha_{i}=q_{i}\right.$, and $p_{i} \uparrow\left[\alpha_{i}, \alpha\right)=p \uparrow$ $\left[\alpha_{i}, \alpha\right)$. 
$i$ is limit. For each $j<i$ consider the sequence $\left\langle p_{k} \uparrow \alpha_{j}: j \leq k<i\right\rangle$. This is a decreasing sequence from $\mathbb{P}_{\alpha_{j}}^{\text {rect }}$ so by the first claim of the Lemma we can form a greatest lower bound $r_{j}$, where $r_{j} \in \mathbb{P}_{\alpha_{j}}^{\text {rect }}$ and $r_{j}$ has height $\sigma=\sup _{k<i} \sigma_{k}$.

It is easy to see that if $m<n$ then $r_{m} \uparrow\left(0, \alpha_{m}\right)=r_{n} \uparrow\left(0, \alpha_{m}\right), r_{m}(0) \uparrow \sigma=$ $r_{n}(0) \uparrow \sigma$, and $r_{m}(0)(\sigma) \subseteq r_{n}(0)(\sigma)$. We define $q_{i}$ such that $\operatorname{dom}\left(q_{i}\right)=\alpha_{i}, q_{i} \uparrow$ $\left(0, \alpha_{j}\right)=r_{j} \uparrow\left(0, \alpha_{j}\right)$ for all $j, q_{i}(0) \uparrow \sigma$ is the common value of $r_{j}(0) \uparrow \sigma$, and $q_{i}(0)(\sigma)=\bigcup_{j<i} r_{j}(0)(\sigma)$.

It is routine to check that $q_{i} \in \mathbb{P}_{\alpha_{i}}^{\text {rect }}$ and $q_{i}$ has height $\sigma$, also that $q_{i} \leq p_{j}\left\lceil\alpha_{i}\right.$ for each $j<i$. Now let $p_{i}\left\lceil\alpha_{i}=q_{i}, p_{i} \uparrow\left[\alpha_{i}, \alpha\right)=p\left\lceil\left[\alpha_{i}, \alpha\right)\right.\right.$.

The construction for the limit step also works for $i=\operatorname{cf}(\alpha)$, and produces $p_{\mathrm{cf}(\alpha)}$ which is in $\mathbb{P}_{\alpha}^{r e c t}$ and refines $p$.

It will be crucial later that we have a certain latitude when we are forming a lower bound for a directed set of conditions. In particular in the argument for Case 4 above we could extend $q_{\operatorname{cf}(\alpha)}(0)(\sigma)$ by adding in $\lambda$ many additional subsets of $\sigma$, and still obtain a lower bound.

\subsection{Iteration and preservation}

Let $V$ be a model of GCH. We describe an iteration $\mathbb{P}_{\infty}^{\diamond}$ of length ON with Easton support to add $\mathrm{a} \diamond_{\mu}$-sequence for every $\mu$ which is inaccessible or the successor of a singular cardinal, and a $\diamond_{\mu}^{+}$-sequence for every $\mu$ which is the successor of a regular cardinal. To do this we begin by forcing with $\mathbb{Q}_{0}^{\diamond}=\operatorname{Add}(\omega, 1)$ and then let $\mathbb{Q}_{\mu}^{\diamond}$ be

1. The poset $\operatorname{Add}(\mu, 1)^{V^{\mathbb{P}_{\mu}}}$ for $\mu$ inaccessible or $\mu$ the successor of a singular cardinal. 2. The poset $\mathbb{Q}^{\diamond}(\mu)^{V^{\mathbb{P}_{\mu}}}$ as described in Sect. 3.1 when $\mu=\lambda^{+}$for $\lambda$ regular.

At all other stages, the forcing is trivial.

We need to show that this iteration $\mathbb{P}_{\infty}^{\diamond}$ preserves all regular instances of supercompactness. Routine arguments show that the iteration preserves all cardinals and cofinalities, together with GCH and the fact that $V^{\mathbb{P}_{\infty}}$ is a model of ZFC. In addition, using the arguments of [1, Lemma 1.1] and [4, Theorem 12.2], it is easily verified that in $V^{\mathbb{P}_{\infty}}, \diamond_{\mu}$ holds for every $\mu$ which is inaccessible or the successor of a singular cardinal, and $\diamond_{\mu}^{+}$holds for every $\mu$ which is the successor of a regular cardinal.

Theorem 5 If $\gamma$ is regular and $\kappa$ is $\gamma$-supercompact in $V$, then $\kappa$ is $\gamma$-supercompact in $V^{\mathbb{P}_{\infty}}$.

Proof It is enough to show that $\kappa$ is $\gamma$-supercompact in $V^{\mathbb{P}_{\gamma+1}^{\diamond}}$, since the rest of the iteration does not change $P_{\kappa} \gamma$. We will distinguish various cases.

$\gamma$ is inaccessible or the successor of a singular cardinal. Here, we give an argument similar to the one presented in [1, Lemma 1.2]. We fix as usual $U$ a supercompactness measure on $P_{\kappa} \gamma$, and $j: V \rightarrow M$ the associated ultrapower map. By GCH we get that $\gamma^{+}<j(\kappa) \leq j(\gamma)<\gamma^{++}$. 
We can factorise $\mathbb{P}_{\gamma+1}^{\diamond}$ as $\mathbb{P}_{\kappa} * \dot{\mathbb{Q}}$, where $\mathbb{Q}$ is the part of the iteration in the interval $[\kappa, \gamma] . \mathbb{Q}$ is a $\kappa$-directed closed forcing of size $\gamma$. Let $G * g$ be the corresponding factorisation of a $\mathbb{P}_{\gamma+1}$-generic filter.

We note that $V[G * g] \vDash \gamma^{\gamma} M[G * g] \subseteq M[G * g]$. Since $|j(\gamma)|=\gamma^{+}$, by the usual arguments, working in $V[G * g]$ we may prolong $G * g$ to $j(G)=G * g * H$ which is $j\left(\mathbb{P}_{\kappa}\right)$-generic, and lift to get $j: V[G] \rightarrow M[G * g * H]$.

Since $V[G * g] \vDash \gamma M[G * g * H] \subseteq M[G * g * H]$, and $j(\mathbb{Q})$ is $j(\kappa)$-directed closed, we may find a lower bound for $j$ " $g$ in $j(\mathbb{Q})$ and use this as a master condition. Since $|j(\gamma)|=\gamma^{+}$we may build in $V[G * g]$ a generic $h$ with $j^{\prime \prime} g \subseteq h$, and finish by lifting to $j: V[G * g] \rightarrow M[G * g * H * h]$.

$\gamma=\delta^{+}$for $\delta$ regular. We fix a supercompactness measure $U$ on $P_{\kappa} \delta^{+}$, and let $j: V \rightarrow M$ be the ultrapower map. As usual $\delta^{++}<j(\kappa)<j\left(\delta^{+}\right)<\delta^{+++}$. Also $j$ is continuous at $\gamma^{+}=\delta^{++}$.

The last step in $\mathbb{P}_{\gamma+1}^{\diamond}$ is $\mathbb{Q}_{\gamma}^{\diamond}$, the forcing for adding a $\diamond_{\delta^{+}}^{+}$-sequence. We will break up $\mathbb{P}_{\gamma+1}^{\diamond}$ as $\mathbb{P}_{\kappa}^{\diamond} * \dot{\mathbb{Q}} * \dot{\mathbb{Q}}_{\gamma}$, where $\mathbb{Q}$ is the iteration in the interval $[\kappa, \gamma)$. We know that $\mathbb{Q}$ is $\kappa$-directed closed and has size at most $\gamma \cdot \mathbb{Q}_{\gamma}^{\diamond}$ is $\gamma$-directed closed and $\gamma^{+}$-cc forcing of size $\gamma^{+}$.

Let $G * g * H$ be the corresponding factorisation of a $\mathbb{P}_{\gamma+1}$-generic filter. Since $G * g$ is generic for forcing of size $\gamma, V[G * g] \vDash \gamma M[G * g] \subseteq M[G * g]$. Since $H$ is generic for $\gamma^{+}$-cc forcing, $V[G * g * H] \vDash \gamma M[G * g * H] \subseteq M[G * g * H]$.

Using this closure, we may as usual prolong $G * g * H$ to $j(G)=G * g * H * h$ which is $j\left(\mathbb{P}_{\kappa}\right)$-generic over $M$, and lift to get $j: V[G] \rightarrow M[j(G)]$. We have that $V[G * g * H] \vDash \gamma M[j(G)] \subseteq M[j(G)]$. Since $|\mathbb{Q}|=\gamma$ and $\mathbb{Q}$ is $\kappa$-directed closed, we may argue just as before to produce $K \supseteq j$ " $g$ which is $j(\mathbb{Q})$-generic and extend once more to $j: V[G * g] \rightarrow M[j(G) * K]$, where $V[G * g * H] \vDash \gamma M[j(G) * K] \subseteq$ $M[j(G) * K]$.

Thus far the argument was fairly routine, but now we need a new idea because $\mathbb{Q}_{\gamma}^{\diamond}$ has size $\gamma^{+}$and is a complicated forcing. We use a version of an idea of Magidor $[11,12]$ with an added twist. We digress briefly to explain how Magidor's technique works in a simpler setting. A more complete explanation of Magidor's method may be found in [2, Corollary 10, pp. 832-833] (see also [3, Lemma 9, pp. 119-120]).

Suppose for a moment that $\mathbb{Q}_{\gamma}$ were the poset $\operatorname{Add}\left(\gamma, \gamma^{+}\right)$. Conditions are partial functions from $\gamma \times \gamma^{+}$to 2 of cardinality less than $\gamma$, ordered by $p \leq q$ if and only if $q \supseteq p$. This is quite similar in the sense that it is $\gamma^{+}$-cc, adds $\gamma^{+}$subsets of $\gamma$ and can be seen as an iteration with $<\gamma$-support.

As usual if $H$ is a generic filter and $\eta<\gamma^{+}$we may form a restricted filter $H \uparrow \eta$ which is generic for $\mathbb{Q}_{\gamma}\left\lceil\eta=\operatorname{Add}(\gamma, \eta)\right.$. For each $\eta<\gamma^{+}$we can form a "partial master condition" $q_{\eta}={ }_{\text {def }} \bigcup j$ “ $H \uparrow \eta$, and the $q_{\eta}$ form a decreasing sequence.

By a standard chain condition argument, if $A$ is a maximal antichain in $\mathbb{Q}_{\gamma}$ then there is $\sigma<\gamma^{+}$such that $A \subseteq \mathbb{Q}_{\gamma}\lceil\eta$ for every $\eta \geq \sigma$. We may now proceed to build a generic filter for $j\left(\mathbb{Q}_{\gamma}\right)$ which is compatible with $H$. We enumerate the maximal antichains in $j\left(\mathbb{Q}_{\gamma}\right)$ in order type $\gamma^{+}$, say as $\left\langle A_{i}: i<\gamma^{+}\right\rangle$.

We now construct a decreasing sequence of conditions $\left\langle r_{i}: i\left\langle\gamma^{+}\right\rangle\right.$, maintaining the hypotheses that $r_{i+1} \in A_{i}$ and $r_{i}$ is compatible with all the $q_{\eta}$. We set $r_{0}$ to be 
the trivial condition, and take unions at limits. Given $r_{i}$ we first find $\eta_{i}$ such that $A_{i} \subseteq\left(j\left(\mathbb{Q}_{\gamma}\right) \uparrow j\left(\eta_{i}\right)\right)$ and $\operatorname{dom}\left(r_{i}\right) \subseteq j(\gamma) \times j\left(\eta_{i}\right)$, which is possible as $j$ is continuous at $\gamma^{+}$. We form $r_{i} \cup q_{\eta_{i}}$, which is a condition because $r_{i}$ is compatible with $q_{\eta_{i}}$, and then use the fact that $A_{i} \subseteq\left(j\left(\mathbb{Q}_{\gamma}\right)\left\lceil j\left(\eta_{i}\right)\right)\right.$ to find $r_{i+1} \leq r_{i} \cup q_{\eta_{i}}$ such that $r_{i+1}$ meets $A_{i}$ and $\operatorname{dom}\left(r_{i+1}\right) \subseteq j(\gamma) \times j\left(\eta_{i}\right)$. The key point is that since $r_{i+1}$ extends $q_{\eta_{i}}$, and $\operatorname{dom}\left(r_{i+1}\right) \subseteq j(\gamma) \times j\left(\eta_{i}\right), r_{i+1}$ is compatible with $q_{\eta}$ for all $\eta$.

Returning to the case at hand, we would like to apply the same idea. But there is a subtle problem, which is that when we form the "partial master conditions" $q_{\eta}$ in the natural way they do not form a decreasing sequence. We now give a detailed analysis of this issue.

We recall that $\mathbb{Q}_{\gamma}$ is an iteration of length $\gamma^{+}$with supports of size $\delta$. On coordinate zero we add $\mathrm{a} \diamond_{\gamma}^{\prime}$ sequence, and then we shoot clubs to make it into a $\diamond_{\gamma}^{+}$-sequence. For $\eta<\gamma^{+}$, let $\mathbb{Q}_{\gamma} \uparrow \eta$ be the iteration up to stage $\eta$ and let $H \uparrow \eta$ be the corresponding generic object.

Since $\mathbb{Q}_{\gamma}^{\diamond}\lceil\eta$ has size $\gamma$, we may compute in $M[j(G) * K]$ the set $j$ " $(H \uparrow \eta)$ and form its "canonical" lower bound $q_{\eta}$ as in Lemma 3.2. We need to analyse the condition $q_{\eta}$ more closely. Let $\zeta=\sup j$ " $\gamma$, so that as usual $\zeta<j(\gamma)$.

$q_{\eta}(0)$ is a sequence of length $\zeta+1$, and $q_{\eta}(0)\lceil\zeta$ is just the union of $j(q)$ as $q$ runs through $H \uparrow 1 . q_{\eta} \uparrow[1, j(\eta))$ is a partial function with support

$$
Z_{\eta}=\bigcup\{j(x): x \in V[G * g], x \subseteq[1, \eta),|x| \leq \delta\}
$$

For each $\alpha \in Z_{\eta}, q_{\eta}(\alpha)$ is a closed set with maximum point $\zeta$, and

$$
q_{\eta}(\alpha) \cap \zeta=\bigcup\{j(q)(\alpha): q \in H \uparrow \eta, \alpha \in j(\operatorname{supp}(q))\}
$$

Recall that $\mathbb{Q}_{\gamma}$ is an iteration in which at stage $\alpha>0$ we are given a set $X_{\alpha} \subseteq \gamma$, and we shoot a club set through the set of places where it is correctly guessed by the generic sequence added at stage 0 . Let $j\left(\left\langle\dot{X}_{\alpha}: \alpha<\gamma^{+}\right\rangle\right)=\left\langle\dot{Y}_{\beta}: \beta<j\left(\gamma^{+}\right)\right\rangle$. For each $q \in H \uparrow \eta$, if $q(0)$ has domain $\mu+1$ then for every $\alpha \in \operatorname{supp}(q), \mu=\max q(\alpha)$ and $q \uparrow \alpha$ determines $\dot{X}_{\alpha} \cap \mu$.

It follows that for every $\alpha \in Z_{\eta}, q_{\eta} \uparrow \alpha$ determines $\dot{Y}_{\alpha} \cap \zeta$, say it forces it to be $\check{y}_{\alpha}$. Then $q_{\eta}(0)(\zeta)$ consists of the sets $y_{\alpha}$ and $q_{\eta}(\alpha) \cap \zeta$ for $\alpha \in Z_{\eta}$. It is easy to see that if $\eta<\eta^{\prime}$ then

1. $q_{\eta^{\prime}} \uparrow[1, j(\eta))=q_{\eta} \uparrow[1, j(\eta))$.

2. $q_{\eta^{\prime}}(0) \uparrow \zeta=q_{\eta}(0) \uparrow \zeta$.

So $q_{\eta^{\prime}}$ is almost an extension of $q_{\eta}$, but not quite because $q_{\eta^{\prime}}(0)$ contains more sets at level $\zeta$.

We need a slightly more detailed analysis of the sets which can appear as $y_{\alpha}$ and $q_{\eta}(\alpha) \cap \zeta$. Fix some $\eta \in\left[\gamma, \gamma^{+}\right)$, and a bijection $\pi \in V$ between $\gamma$ and $\eta$. We may view $H \uparrow[1, \eta)$ as giving us a sequence $\left\langle C_{\alpha}: 0<\alpha<\eta\right\rangle$ of club subsets of $\gamma$, which we may then code up as $W \subseteq \eta \times \gamma$, where $(\alpha, i) \in W \leftrightarrow i \in C_{\alpha}$. Define $\bar{W} \subseteq \gamma \times \gamma$ by $(\alpha, i) \in \bar{W} \leftrightarrow(\pi(\alpha), i) \in W$. It is now routine to check that if we define $W^{*}=\bigcup_{i<\gamma} j(\bar{W} \cap(i \times i))$, then $W^{*} \subseteq \zeta \times \zeta$ and the sets which appear in 
the form $\left\{v<\zeta:(\alpha, v) \in W^{*}\right\}$ as $\alpha$ runs through $\zeta$ are precisely the sets $q_{\eta}(\alpha) \cap \zeta$ for $\alpha \in Z_{\eta}$. A similar analysis works for the $y_{\alpha}$, with the sequence $\left\langle X_{\alpha}: 0<\alpha<\eta\right\rangle$ in place of $\left\langle C_{\alpha}: 0<\alpha<\eta\right\rangle$.

Now let $T \subseteq \gamma \times \gamma$ with $T \in V[G * g]$ being arbitrary. Clearly, since $V[G * g * H] \vDash$ $\gamma_{M}[j(G) * K] \subseteq M[j(G) * K], T \in M[j(G) * K]$. We claim that the map which takes such $T$ to $T^{\dagger}=\bigcup_{i<\gamma} j(T \cap(i \times i))$ is in $M[j(G) * K]$. To prove this claim we split into several cases depending on the nature of $\delta$.

Case $1 \delta$ is inaccessible. In this case $\mathbb{Q}_{\delta}^{\diamond}$ is $\operatorname{Add}(\delta, 1)$, so $\mathbb{P}_{\gamma}$ is a forcing poset of size $\delta$. So for each $i<\gamma$ the $\mathbb{P}_{\gamma}$ names for subsets of $i \times i$ are essentially subsets of $\delta$, and we know that $j\lceil\wp(\delta) \in M$.

Case $2 \delta$ is the successor of a regular cardinal. Then $\mathbb{Q}_{\delta}^{\diamond}$ is an iteration of length $\delta^{+}$ with $\delta^{+}$-cc, so that all bounded subsets of $\gamma$ appear in the extension by some initial segment, a forcing poset of size $\delta$. So again all the relevant names are essentially subsets of $\delta$ and we are done as in Case 1 .

Case $3 \delta$ is the successor of a singular cardinal $\rho$. The cardinality of $\mathbb{P}_{\rho}^{\diamond}$ is $\delta$, we are doing Cohen forcing at $\delta$, so $\mathbb{P}_{\gamma}^{\diamond}$ has size $\delta$, and we are done as in Case 1.

So now we can compute in $M[j(G) * K]$ the set $P$ of all $T^{\dagger}$ as above. This set has size bounded by $j(\delta)$ so we can augment each of the $q_{\eta}$ 's defined above by adding all elements of $P$ to coordinate zero at level $\zeta$. This gives us a new sequence of partial master conditions $p_{\eta}^{*}$ with the crucial extra property that the $p_{\eta}^{*}$ are literally decreasing. We may now proceed with Magidor's argument exactly as above.

\section{The proof of the main theorem}

Having completed the discussion found in Sects. 1-3, we are now ready to turn our attention to the proof of Theorem 2 .

Proof Let $V \vDash$ "ZFC $+\mathrm{GCH}+\mathcal{K} \neq \emptyset$ is the class of supercompact cardinals". We begin by forcing with the poset $\mathbb{P}_{\infty}^{\square}$ of Sect. 2 , to obtain the extension $V_{1}=V^{\mathbb{P}} \square$. By Theorem 4 , we know that all $V$-supercompact cardinals are preserved to $V_{1}$. In addition, as in Sect. 2, we may write $\mathbb{P}_{\infty}^{\square}=\mathbb{Q} * \dot{\mathbb{R}}$, where $|\mathbb{Q}|=\omega, \mathbb{Q}$ is nontrivial, and $\vdash_{\mathbb{Q}}$ “ $\mathbb{R}$ is $(\omega+1)$-strategically closed". By Theorem 3, this means that any cardinal supercompact in $V_{1}$ had to have been supercompact in $V$. Thus, $\mathcal{K}$ remains the class of supercompact cardinals in $V_{1}$. Also, by the work of Sect. 2, we know that $\square_{\gamma}^{S}$ holds in $V_{1}$, for every infinite cardinal $\gamma$ and $S=\operatorname{Safe}(\gamma)$, and that $\mathrm{GCH}$ is preserved to $V_{1}$ as well.

We force now over $V_{1}$ with the poset of [3], which for convenience we denote here by $\mathbb{P}_{\infty}^{L B L}$. This preserves all $V_{1}$-instances of supercompactness, $\mathrm{GCH}$, and all cardinals and cofinalities, and in addition forces level by level equivalence between strong compactness and supercompactness. Call the resulting extension $V_{2}$. Since $\mathbb{P}_{\infty}^{L B L}$ may be defined so as to admit a closure point at $\omega$ (see [3] for further details), Theorem 3 and the remarks immediately following imply that $\mathcal{K}$ remains the class 
of supercompact cardinals in $V_{2}$. Further, by the upwards absoluteness of any form of square in a cardinal preserving forcing extension (see the discussion given in the proof of Theorem 1 of [1]) and the remarks in the paragraph following the statement of Definition 2.2, $\square_{\gamma}^{S}$ for $S=\operatorname{Safe}(\gamma)$ remains true in $V_{2}$ for all infinite cardinals $\gamma$.

Finally, we force over $V_{2}$ with the poset $\mathbb{P}_{\infty}^{\diamond}$ of Sect. 3 , to obtain the extension $V_{3}$. By the work of Sect. 3, we know that in $V_{3}, \diamond_{\mu}$ holds for every $\mu$ which is inaccessible or the successor of a singular cardinal, and $\diamond_{\mu}^{+}$holds for every $\mu$ which is the successor of a regular cardinal. GCH is preserved to $V_{3}$ as well. By Theorem 5, we also know that all $V_{2}$-supercompact cardinals are preserved to $V_{3}$. In addition, we may write $\mathbb{P}_{\infty}^{\diamond}=\mathbb{Q}^{\prime} * \dot{\mathbb{R}}^{\prime}$, where $\left|\mathbb{Q}^{\prime}\right|=\omega, \mathbb{Q}^{\prime}$ is nontrivial, and $\Vdash_{\mathbb{Q}^{\prime}}$ ' $\dot{\mathbb{R}}^{\prime}$ is $(\omega+1)$-strategically closed". By Theorem 3, this means that any cardinal supercompact in $V_{3}$ had to have been supercompact in $V_{2}$. Thus, $\mathcal{K}$ remains the class of supercompact cardinals in $V_{3}$. Also, as in the last sentence of the preceding paragraph, $\square_{\gamma}^{S}$ for $S=\operatorname{Safe}(\gamma)$ remains true in $V_{3}$ for all infinite cardinals $\gamma$.

The proof of Theorem 2 is now completed by the following lemma.

Lemma 4.1 $V_{3} \vDash$ "Level by level equivalence between strong compactness and supercompactness holds".

Proof We mimic the proof of Lemma 1.3 of [1]. Suppose $V_{3} \vDash$ " $\kappa<\lambda$ are regular cardinals such that $\kappa$ is $\lambda$-strongly compact and $\kappa$ isn't a measurable limit of cardinals $\delta$ which are $\lambda$-supercompact". By Theorem 5, any cardinal $\delta$ such that $\delta$ is $\lambda$-supercompact in $V_{2}$ remains $\lambda$-supercompact in $V_{3}$. We may therefore infer that $V_{2} \vDash$ “ $\kappa<\lambda$ are regular cardinals such that $\kappa$ isn't a measurable limit of cardinals $\delta$ which are $\lambda$-supercompact".

By the definition of $\mathbb{P}_{\infty}$, it is easily seen that $\mathbb{P}_{\infty}$ is mild with respect to $\kappa$. Hence, by the factorisation of $\mathbb{P}_{\infty}^{\diamond}$ given above and Theorem $3, V_{2} \vDash$ " $\kappa$ is $\lambda$-strongly compact". Consequently, by level by level equivalence between strong compactness and supercompactness in $V_{2}, V_{2} \vDash$ " $\kappa$ is $\lambda$-supercompact", so another application of Theorem 5 yields that $V_{3} \vDash$ " $\kappa$ is $\lambda$-supercompact". This completes the proof of Lemma 4.1.

By defining $\mathbb{P}=\mathbb{P}_{\infty}^{\square} * \dot{\mathbb{P}}_{\infty}^{L B L} * \dot{\mathbb{P}}_{\infty}^{\diamond}$, the proof of Theorem 2 is now complete.

We conclude by asking if the results of Theorem 2 can be generalised further. In particular, is it possible to extend the above techniques so that $\diamond_{\mu}^{+}$holds for every successor cardinal $\mu$, and not just successors of regular cardinals?

\section{References}

1. Apter, A.: Diamond, square, and level by level equivalence. Arch. Math. Log. 44, 387-395 (2005)

2. Apter, A., Hamkins, J.D.: Indestructibility and the level-by-level agreement between strong compactness and supercompactness. J. Symb. Log. 67, 820-840 (2002)

3. Apter, A., Shelah, S.: On the strong equality between supercompactness and strong compactness. Trans. Am. Math. Soc. 349, 103-128 (1997)

4. Cummings, J., Foreman, M., Magidor, M.: Squares, scales, and stationary reflection. J. Math. Log. 1, 35-98 (2001)

5. Devlin, K.: Constructibility, Perspectives in Mathematical Logic. Springer, Berlin (1984)

6. Devlin, K., Johnsbräten, H.: The Souslin problem. Lecture Notes in Mathematics, vol. 405. Springer, Berlin-New York (1974) 
7. Foreman, M., Magidor, M.: A very weak square principle. J. Symb. Log. 62, 175-196 (1997)

8. Hamkins, J.D.: Extensions with the approximation and cover properties have no new large cardinals. Fundam. Math. 180, 257-277 (2003)

9. Hamkins, J.D.: Gap forcing. Isr. J. Math. 125, 237-252 (2001)

10. Hamkins, J.D.: Gap forcing: generalizing the Lévy-Solovay theorem. Bull. Symb. Log. 5, 264-272 (1999)

11. Jech, T., Magidor, M., Mitchell, W., Prikry, K.: Precipitous ideals. J. Symb. Log. 45, 1-8 (1980)

12. Magidor, M.: On the existence of nonregular ultrafilters and the cardinality of ultrapowers. Trans. Am. Math. Soc. 249, 97-111 (1979)

13. Menas, T.: On strong compactness and supercompactness. Ann. Math. Log. 7, 327-359 (1974)

14. Solovay, R., Reinhardt, W., Kanamori, A.: Strong axioms of infinity and elementary embeddings. Ann. Math. Log. 13, 73-116 (1978) 